\title{
EVOLUTION OF ENTERPRISE ARCHITECTURE DISCIPLINE Towards a Unified Developing Theory of Enterprise Architecture Body of Knowledge as an Evolving Discipline
}

\author{
Hadi Kandjani and Peter Bernus \\ Centre for Enterprise Architecture Research and Management (CEARM) \\ School of ICT, Griffith University, Brisbane, Australia \\ \{H.Kandjani, P.Bernus\}@griffith.edu.au
}

Keywords: Enterprise Architecture Discipline, Unified Theory, Viable System Model, Co-evolution Path Model, Enterprise Architecture Cybernetics

\begin{abstract}
When studying enterprises as complex systems through the Enterprise Architecture (EA) discipline, researchers not only apply models, methods and theories of management and control - they 'should' also use the same from engineering, linguistics, cognitive science, environmental science, biology, social science, artificial intelligence, systems thinking and cybernetics. This diversity of related disciplines derives from the nature of enterprises as multi-faceted, multi-disciplinary entities with interacting dimensions and different design- and evolution concerns. We believe that for the EA discipline (EAd), like any other developing and evolving discipline, there should exist a unified terminology, models and methodology. There already exists a fundamental and generalised theory of EAd, GERAM, however, it is a minimalist theory, not prescribing any particular reference models or any concrete methodology. Therefore, practitioners developed particular frameworks, adding concrete methodologies / reference models specific to the domain / type of change to tackle. The question is: is possible to extend the EA Body of Knowledge (EABOK) with common elements - independent from the domain / type of change? In other words, what is a unified evolving theory of EAd? To model the discipline-as-a-system, we use Beer's Viable System Model (VSM) and introduce three basic components of EAd as a viable system. A 'co-evolution mechanisms' for EAd is proposed, and a cybernetic model of co-evolution applied to EAd. We also discuss a cybernetic model of EAd using Checkland's model for discipline development.
\end{abstract}

\section{INTRODUCTION}

For Enterprise Architecture (EA), like any other developing and evolving discipline, there should exist a theory, with terminology and rules capable of unifying the constituent models and methodologies developed by contributing disciplines. There already exists a fundamental theory of EA: GERAM, however it is a minimalist theory, not prescribing any particular reference models or methodology (IFIP-IFAC-Task-Force, 1999; ISO 15704 2000, Amd. 2005).

The GERAM framework includes a terminology, with the concepts of enterprise-entity, life cycle, life history, modelling languages, models, instantiation and tools, and rules of life cycle relationships, conceived for use in the management of any change. The framework provides the user with a generic reference model for constituents of a life cycle and a modelling framework, with complete subdivision of view point concepts (NB these are called 'views' in the original document, but for compatibility with ISO 42010 (2011) we use the term viewpoint). Note that this architecture framework and its theory are independent from the domain and type of change.

For pragmatic purposes, practitioners who develop(ed) particular architectural frameworks add methodologies and/or reference models specific to the application domain and/or the type of change for which they are intended to be applicable.

For example, DoDAF (2009), as a particular architectural framework, includes an interoperability capability assessment reference model, defined as a series of levels, called 'Levels of Information System Interoperability’ (LISI). 
TOGAF (1999-2011), as a particular architecture framework, comes with a methodology to develop IT architecture, called the 'Architecture Development Method' (ADM).

These particular frameworks (DoDAF, TOGAF etc.) are domain dependent and were developed for a specific type of change; whereupon, general architectural frameworks, such as GERAM, are independent from the domain and type of change.

The critical question in this paper is that: how is it possible to extend the EA Body of Knowledge with common elements that are domain independent as well as independent from the type of change? In other words: what is a unified evolving model of EA Body of Knowledge? By answering this question, we could in fact have an extension of the theory to the architecture of any large scale complex system.

Cybernetics and General Systems Theory (GST) have previously attacked these types of problems at the same, or similar, level of abstraction and generality. Therefore, to develop and extend the EA discipline we need to incorporate the apport of previously related disciplines and their theories into a unified theory. As this will no doubt be a long term process we must treat EA as an evolving and developing discipline.

Norbert Wiener defined cybernetics as "the science of control and communication in the animal and machine" (Wiener 1948). Ashby (1956) also calls cybernetics the art of "steermanship" which studies co-ordination, regulation and control of systems, arguing that the "truths of cybernetics are not conditional on their being derived from some other branch of science". Therefore the field embraces a set of self-contained groundings and foundations, which Ashby tried to describe in his book (ibid). He addressed the complexity of a system as one of the peculiarities of cybernetics and indicated that cybernetics prescribes a scientific method of dealing with complexity as a critical attribute of a system.

Stafford Beer believed that the dynamics of enterprises is about "the manipulation of men, material, machinery and money: the four Ms", plus an even more fundamental "manipulation" (from microscopic biological organisms to large scale systems, including enterprises): the "management of complexity” (Beer 1966; 1985).

Enterprises are best understood as intrinsically complex adaptive living systems: they can not purely be considered as 'designed systems', as deliberate design/control episodes and processes ('enterprise engineering' using design models) are intermixed with emergent change episodes and processes (that may perhaps be explained by models). The mix of deliberate and emerging processes can create a situation in which the enterprise as a system is in a dynamic equilibrium (for some stretch of time) - a property studied in General Systems Theory (Boulding, 1956; Bertalanffy 1968). The evolution of the enterprise (or enterprises, networks, industries, the economy, society, etc) includes emergent as well as the deliberate aspects of system change, therefore an EA theory must interpret previous research in both.

This unified theory is indeed to be a developing theory, describing evolution of the EA body of knowledge, therefore it should remain open for further continuous contributions of EA practitioners and researchers. The integrating, or interdisciplinary, aspect of EA manifests when studying enterprises as complex systems. Here, researchers not only apply models, methods and theories of management and control (and apply the same from engineering, linguistics, cognitive science, environmental science, biology, social science, artificial intelligence, systems thinking and cybernetics), there needs to be a synthesis of these.

Given this standpoint many theoreticians can contribute to the development of a unified theory of designing / architecting complex systems, taking into account a list of concerns expressed (issues addressed) by different disciplines that are related to 'designing' systems. We call these design- or architecture- concerns 'metaphors'. We can describe the architecture (i.e. 'architecting') process as:

- a Conversation between the controller of the system, the system's 'operations' and the controllers of environmental 'entities' (Conversation Theory (Pask, 1975)),

- a Decisional \& Resource Allocation Process (using GRAI Grid (Doumeingts, 1984; 1998)),

- Complex Process managed to reduce complexity and improve the likelihood of success (applying Axiomatic Design Theory (Suh, 1990; 2001; 2005)),

- an Emergent and Evolutionary Process (using Complex Adaptive Systems Theory (Holland 1992; Gell-Mann 1994)),

- a Planning \& Prediction Process (using Multi-Agent Systems Theory theories (Wooldridge and Jennings, 1995; Wooldridge, 2002)),

- a Participatory Process (using models of Participatory Design (Kensing, Simonsen, \& Bødker, 1998; Bødker, Kensing, \& Simonsen, 2004)), 
- a Change Process (using Re-engineering Methods and approaches (Hammer \& Stanton, 1995)), and

- a Learning Process (using Systems thinking and Cybernetics theories (Ashby, 1956; 1960; Senge, 1993; Nonaka and Takeuchi, 1995).

To develop a unified theory of large scale systems evolution as an extension to the EA body of knowledge, one should therefore review previously developed theories, models and terminologies that study the problem of designing / architecting complex systems.

In order to ensure that this unified theory has sufficient breadth and depth, it is useful to analyse how researchers previously considered the problems and concerns of this area. This useful to understand underlying concerns and problems that various researchers have had when designing architectural processes/ frameworks, but it could also bring new discoveries via this theory unification process.

When studying enterprises as (partially designed and partially evolving) complex evolving systems, many researchers and practitioners implicitly apply methods and models derived from laws and theories of systems thinking and cybernetics. Cybernetics, as an interdisciplinary movement, has formulated multiple laws and theories of complex systems, but each one is presented on a different level of formality, generality and abstraction. Consequently, the application of these laws and theories in Enterprise Architecture (EA) also lack harmony. Therefore, we introduce EA Cybernetics as a field of EA with the intention to harmonise, formalise, synthesise and systematise results of multiple disciplines, using systems thinking and cybernetics, for a concerted and coherent application.

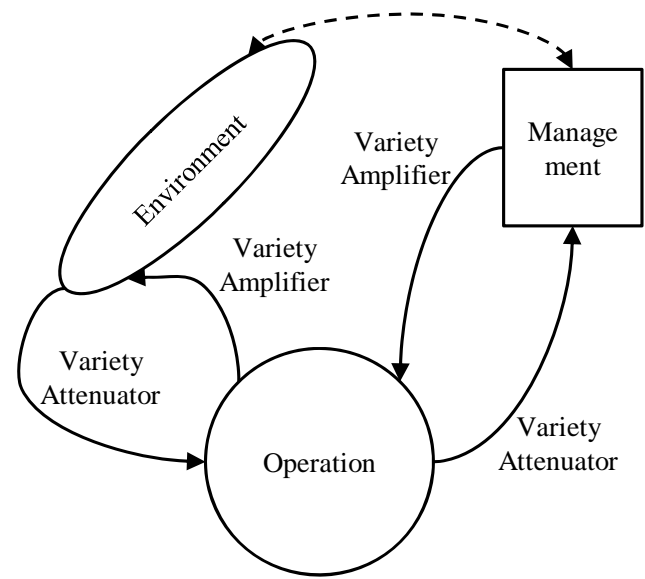

Figure 1. Three components of the Viable System Model (VSM) (Beer, 1985)
EA Cybernetics is the re-interpretation of old- and new theories to understand their individual contributions, and to point at the need for genuinely new results when designing and creating complex systems. Cybernetic thinking is a way to unify / relate the apport of multiple disciplines as the explain the 'architecting process'. Such a synthesis would be the source of a new, unified theory of EA, giving rise to more powerful theories, methodologies and reference models than available today.

\section{VIABILITY OF THE EA DISCIPLINE AND EFFECTIVE EA PRACTICE}

In this section we propose a viable model of EA as an interdisciplinary discipline of designing, creating and maintaining complex systems.

\subsection{Beer's Viable System Model}

Beer (1979) describes every system as consisting of three main interacting components: Management, Operation and Environment (see Fig.1).

Every system of interest (circle in the figure) has a meta-system as its management (represented as a square in the figure) and operates in an environment (represented as an oval shape in the figure), where each component could be further decomposed into more detailed elements. There are communication channels among these three components to keep the operation in homeostasis: these channels are called 'variety attenuators' and 'variety amplifiers' (Beer 1979; Beer 1981; Beer 1985).

According to Beer (1979) the 'variety' of the operations is always less than that of the environment, and the 'variety of management' is always less than the variety of operations. In contrast, based on Ashby's law of requisite variety (1956), in order to achieve dynamic stability under change, the variety of operations should be equal to that of its relevant environment, and the variety of management should be at least equal to that of operations. In fact variety attenuation and amplification mechanisms need to be designed in order to keep the system of interest viable ('evolvable') in its environment.

However, sometimes the enterprise's mission, instead of viability and 'eternality' of the enterprise, is a temporary existence, therefore the demolition or deconstruction of enterprise or enterprise entities is an equally important aspect to consider. Like in 
construction and civil engineering, a demolition plan is a set of processes to tear down buildings or other structures. The same concept applies in EA.

For this purpose, a cybernetic model of EAd must cover the complete life history of the enterprises as systems (and system of systems), including all significant life events - creation, reproduction, merger, as well as decommissioning / end of life. It is especially this last one, end of life, that has received insufficient attention in literature, therefore we propose the concept of Fatal System Model (FSM) stressing that EA should address the cradle-to-grave aspect of the enterprise, from birth (creation of enterprises or agglomerations thereof) to decommissioning (states which finally make the enterprise collapse or dissolve, perhaps preserving valuable elements for re-use).

\subsection{The EA Discipline as a Viable System}

It is possible to map the three components of Beer's VSM to the EA Discipline itself, and to its surrounding environment. We consider the enterprise-related disciplines as ‘operation' shown as a circle, and the EA discipline as its integrating and interdisciplinary meta-system ('management') shown as a square, with EA's task being to observe and cross-fertilise enterprise problem domains as well as to observe the 'environment' (Fig.2).

There are communication channels (acting as variety attenuators and amplifiers) among the three components to achieve / maintain the requisite variety, i.e. in order to keep the EA discipline and its related disciplines as a system in homeostasis.

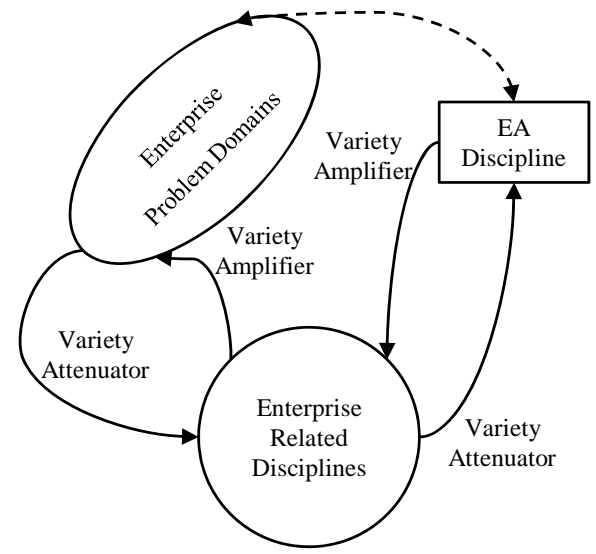

Figure 2. Three components of a Viable EA Discipline

The EA discipline acts as a meta-system that investigates the enterprise problem domains and using attenuation mechanism, invokes the relevant terminology, models and theories from enterpriserelated disciplines (e.g. systems thinking and cybernetics, industrial engineering, management science, control engineering, information and communication technology) to respond to new issues arising in enterprise problem domains.

Changes in the problem domains mandate the evolution of individual enterprise related disciplines, so as to respond to the new requirements of the evolving environment. In fact the evolution of enterprise-related disciplines and enterprise problem domains are coupled and mutually dependent, and the EA Discipline should act as a meta-system/ management system regulating the requisite variety between operations and the environment.

In order to harmonise this co-evolution, we need to understand what are the relevant mechanisms to guarantee an effective evolution of EA itself

If we consider EA as 'problem solving', then the step by step stages of co-evolution would be: 1) diagnose a significant problem in the enterprise problem domain, 2) invoke one or more relevant disciplines studying the enterprise problem domain and decide if such multi-disciplinary combined action is adequate, and if not, then 3) provide solutions for enterprise problems by harmonising and integrating multiple theories, models, techniques and methods from relevant disciplines in a synthesis (new or extended theory), and 4) adopt any 'new' case records of relevant disciplines and mutual contributions of EA and relevant disciplines into the EA Body of Knowledge.

The need for a unifying theory clarifies the role of EA as a meta-system (as in Beer's VSM) that answers the question: a) what enterprise problems domains would be (or should be) addressed in a specific EA practices?, b) what would be the invoked disciplines targeting the problem domains to solve the problem in combined use?, c) how to formalise and harmonise other disciplines' contributions and apply them in an EA practice?

As the invoked disciples are continuously progressing and evolving in their specific domain and field of application, a more effective EA practice could be guaranteed if the evolution of these disciplines were influences or monitored by the EA discipline and the findings reflected in EA theory and practice when necessary.

We discussed three components of a viable EA discipline so far, now the question may arise: what are the mechanisms to keep the requisite variety of the EA discipline and maintain it as a viable system? 


\section{CO-EVOLUTION MECHANISMS FOR AN EVOLVING EA DISCIPLINE}

We discussed three components of a viable EA discipline in Section 2, now the question arises: what are the mechanisms to keep the requisite variety of the EA discipline as a viable system?

\subsection{Co-evolution Path Model, Dynamic Homeostasis vs. Dynamic Hetereostasis}

Beer (1966) argues that a key property of a viable system and a "measure of its submission to the control mechanism" is its ability to maintain its equilibrium or homeostasis, which he defines as “constancy of some critical variables (outputs)". In our model of co-evolution, we define the dynamic sustenance of requisite variety based on Ashby's law: "only variety can destroy variety" (Ashby 1956), paraphrased by Beer (1979) as "variety absorbs variety".

Here, 'variety' is the number of possible states of a system (Beer 1981), or as recently re-interpreted and refined by Kandjani and Bernus (2011), the number of relevant states of a system.

For a system to dynamically achieve / maintain requisite variety and to be in dynamic equilibrium, the system requires communication channels and feedback loops. These channels serve as selfperpetuating mechanism and include both attenuation and amplification mechanisms. (Note that for the discussion below what we call a 'system' includes the system's controller.)

Considering the system and its environment as two coupled entities, if one component is perturbed, the effect of that perturbation on the other component is either amplified through positive feedback, or may be reversed (attenuated) through negative feedback.

The role of the negative feedback loop is to reverse the effect of the initial perturbation and restore the system's homeostasis (in which critical variables are stable), while positive feedback can create unstable states (Ashby 1940).

We observe that both a system and its environment (including systems in that environment) evolve, and the change can create imbalance between the requisite variety (maintained by the controller) of our system of interest and the variety that would be required for it to maintain homeostasis. In other words, systems that want to live long must co-evolve with their environment.

More formally: we consider the environment an entity with a possible set of observable states and if two such states require different response from the system then the system must be able to differentiate between them (thus they are two different relevant states) . (Note that we may not necessarily be able to describe the environment as a system, although it may contain one or more systems.)

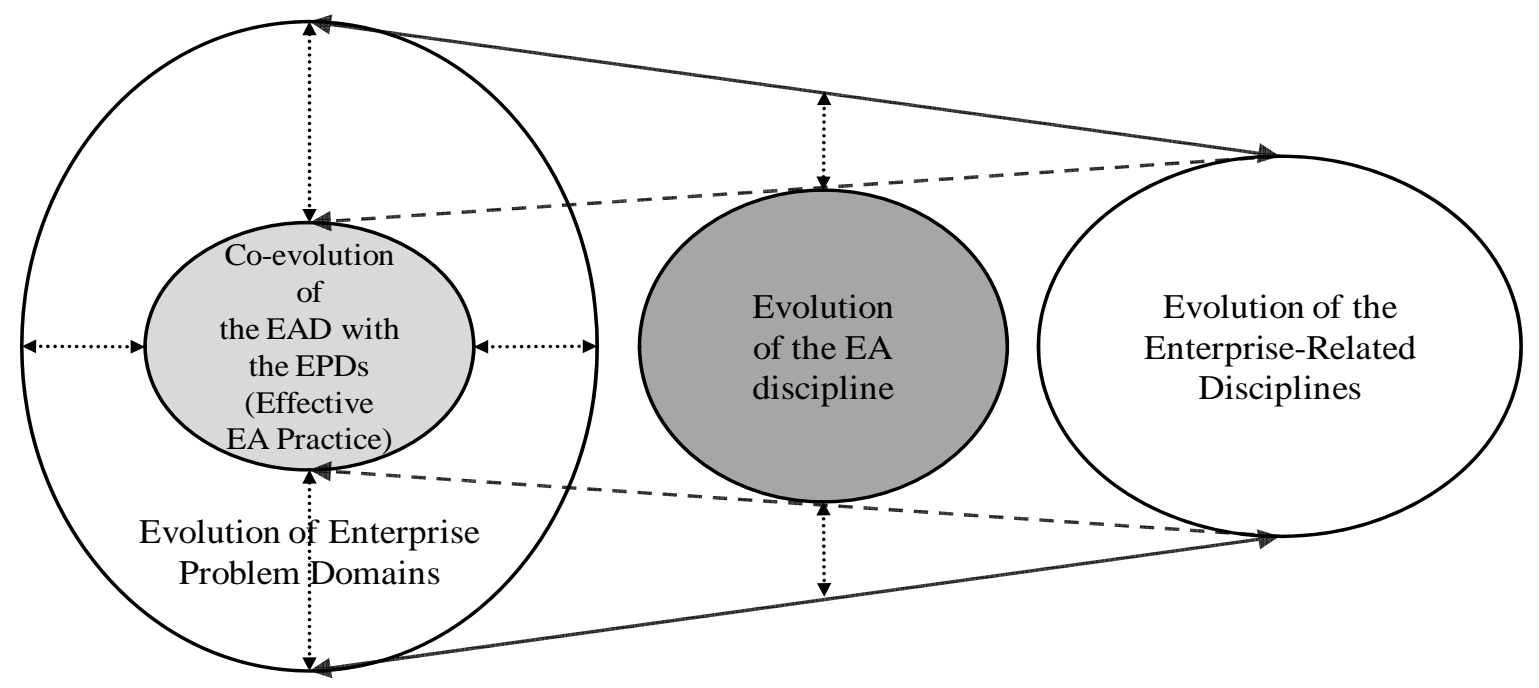

Figure 3. An effective EA Practice: Co-evolution of EA Discipline with Enterprise Problem Domains through invocation of relevant theories, models and methods from Enterprise-Related Disciplines 
Consequently, in Fig. 4, the complexity of a system $\left(\mathrm{C}_{S}\right)$ is defined to be the complexity of the model that the controller of the system maintains (appears to be maintaining) in order to manage the system's operations, and to maintain adequate interaction with the environment.

The complexity of the system's environment $\left(C_{E}\right)$ is a relative notion and is defined to be the complexity of the model of the environment that the controller of the system would need to maintain the system's homeostasis (although, yet again, it is sufficient if, in the eyes of an external observer, the system's controller appears to be maintaining such model). Specifically, such an 'environment model' must have predictive capability, so that the system, while interoperating with the environment, can maintain a homeostatic trajectory in time (and space).

An environment model would include a) models of external systems (including models of their controllers and operations), and b) a model of the rest of the environment.

These models are needed to be able to represent and predict the states of signals and resources among the system, the external systems and the rest of the environment. This because based on the theorem of the 'Good Regulator' (Conant and Ashby 1970), a good controller of a system must have a model of that system with an equal complexity at its disposal as the system to be controlled has.

Notice (Fig.4) that 1) If the complexity of the system (CS) equals to that of its environment (CE), then the system has the requisite variety and is in static equilibrium. However, any change in the complexity of the environment should be sensed by the system's self-perpetuating mechanism to restore the system to its initial state or to create a new equilibrium state; 2) If the complexity of the environment is greater than that of the system, then the system should attenuate the effects of this complexity, i.e., change and co-evolve with its environment (in other words, the environment produced, or is recognised to have the potential to produce, some states in which the system can not function adequately); 3) If the complexity of the system is greater than that of its environment, then the system can potentially create a set of different states and perform behaviours which are not differentiated by its environment. The system can identify this extra complexity as undesired, or use an amplification mechanism to create new differentiations in the environment (e.g. marketing of new goods / services).

\section{Co-evolution}

of the System and its Environment through First and Second Feedback loops

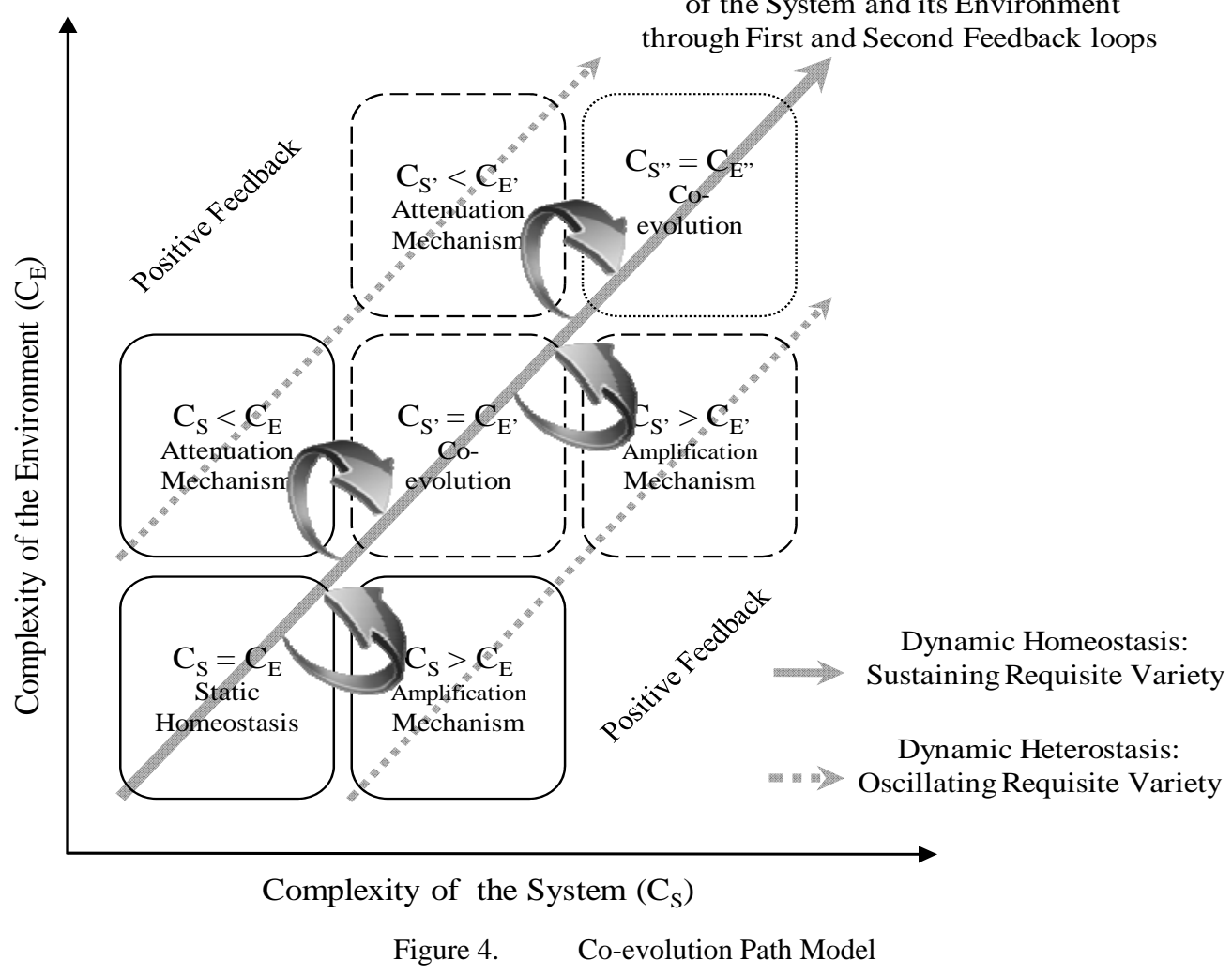


Enterprises as 'live systems' have a number of variables characterising essential survival properties. Ashby (1960) refers to these as 'essential variables' (crucial to a system's survival) - modern literature would refer to these as critical success factors measured by strategic 'key performance indicators'. Ashby (1960) defines survival as: “... a line of behaviour [that] takes no essential variable outside given limits" (Ashby 1960; Geoghegan and Pangaro 2009). Therefore, by definition, any line of behaviour outside limits of essential variables is on the non-viable system path and is fatal to the system's lifeline.

For a system to be regarded as adaptive, and therefore viable, Ashby introduces two necessary feedback loops (Ashby 1960; Geoghegan and Pangaro 2009; Umpleby 2009). The first, frequently operating, feedback loop makes small parametric modifications and corrections to the system. As opposed to this, the second loop changes the structure or architecture of the system and operates if the tolerance of essential is predicted to fall outside the limits of survival. If the system's second feedback loop does not respond to the changes in complexity of the environment, then the system will be on a non-viable path.

Based on Ashby's theory of adaptation (1960), Umpleby (2009) indicates that the first feedback loop is necessary for a system to learn a pattern of behaviour necessary for a specific environment, while the second feedback loop is required for a system to identify the changes in the environment and design and create new patterns of behaviour.

If there is a dramatic increase in complexity of the environment on which the system is not prepared to act (due to scarcity of resources, lack of dynamic capability, inability to create new structures / adapt its architecture in a timely manner), then the lack of an appropriate second feedback loop makes the system non-viable and the system is doomed to fail.

\subsection{Co-evolution Path Model of the EA Discipline}

Looking at EA as a system (the 'discipline-as-asystem') the co-evolution model of Section 3.1 applies to that system too, therefeore the question: what are the co-evolution mechanisms through which the EA discipline can maintain its requisite variety to remain relevant in light of changes to evolving enterprise problem domains?
EA as an integrating discipline invokes models, theories, and methods of related disciplines, an effective co-evolution is only guaranteed by:

a) invoking the right theories, models, and methods from Enterprise related Disciplines (ERD) to address new and emerging Enterprise Problem Domains (EPD) in a combined use (attenuation mechanism), and

b) promoting new synthesised EA terminologies, reference models, and methods to provide solutions in enterprise problems domains using a holistic approach (amplification mechanism).

Thus, if at any one time the variety of the unified EA theory is less than the variety of the enterprise problem domains, then EA can not respond to the evolution of enterprise and enterprise architecture as a discipline must increase its variety by attenuating the relevant variety through adopting new elements from relevant enterprise related disciplines.

On the other hand, an Enterprise Architect should also formulate and execute a promotion mechanism if the variety of EA models methods and frameworks is more than the variety of the enterprise problem domains. In this case, system managers, users, and stakeholders would not be able to comprehend these complex EA models, methods and etc. and would probably avoid using them in the evolution of enterprise; therefore an enterprise architect should decrease the variety of its models by amplifying the variety of the models, or promote the use of more complex models to invent solutions for the enterprise's extended 'new action domains'.

By using these mechanisms (invocation and promotion), it would be possible to sustain the coevolution of the EA discipline and of problem domains, to ensure that EA is adaptively and effectively addressing issues of its problem domains.

The evolution of enterprise related disciplines should therefore be closely monitored so as to be able to perform the mentioned 'invocation' and 'promotion' to provide enterprise problem domains with relevant combined discipline-contributions in any EA practice (Fig.5).

\section{CYBERNETIC MODEL OF EA AS AN EVOLVING \& DEVELOPING DISCIPLINE}

Enterprise Architecture, like any other developing discipline, needs a model for theory development, theory testing and knowledge creation. Anderton and Checkland (1977) developed a model of any 
developing discipline to demonstrate the cyclic interaction between theory development and formulation for a problem, and theory testing (Anderton and Checkland 1977; Checkland 1996).

For EA to be a developing discipline (Fig.6), we consider the real world enterprise problem domains as the source of the development process that give rise to issues that are addressed by theories, models and methods in enterprise related disciplines. These will shape ideas by which two types of theories could be developed (Checkland 1996):

a) substantive theories derived from related disciplines to apply relevant models, theories and methods in enterprise problem domains, and

b) methodological theories about how to individually apply enterprise related disciplines in enterprise problem domains.

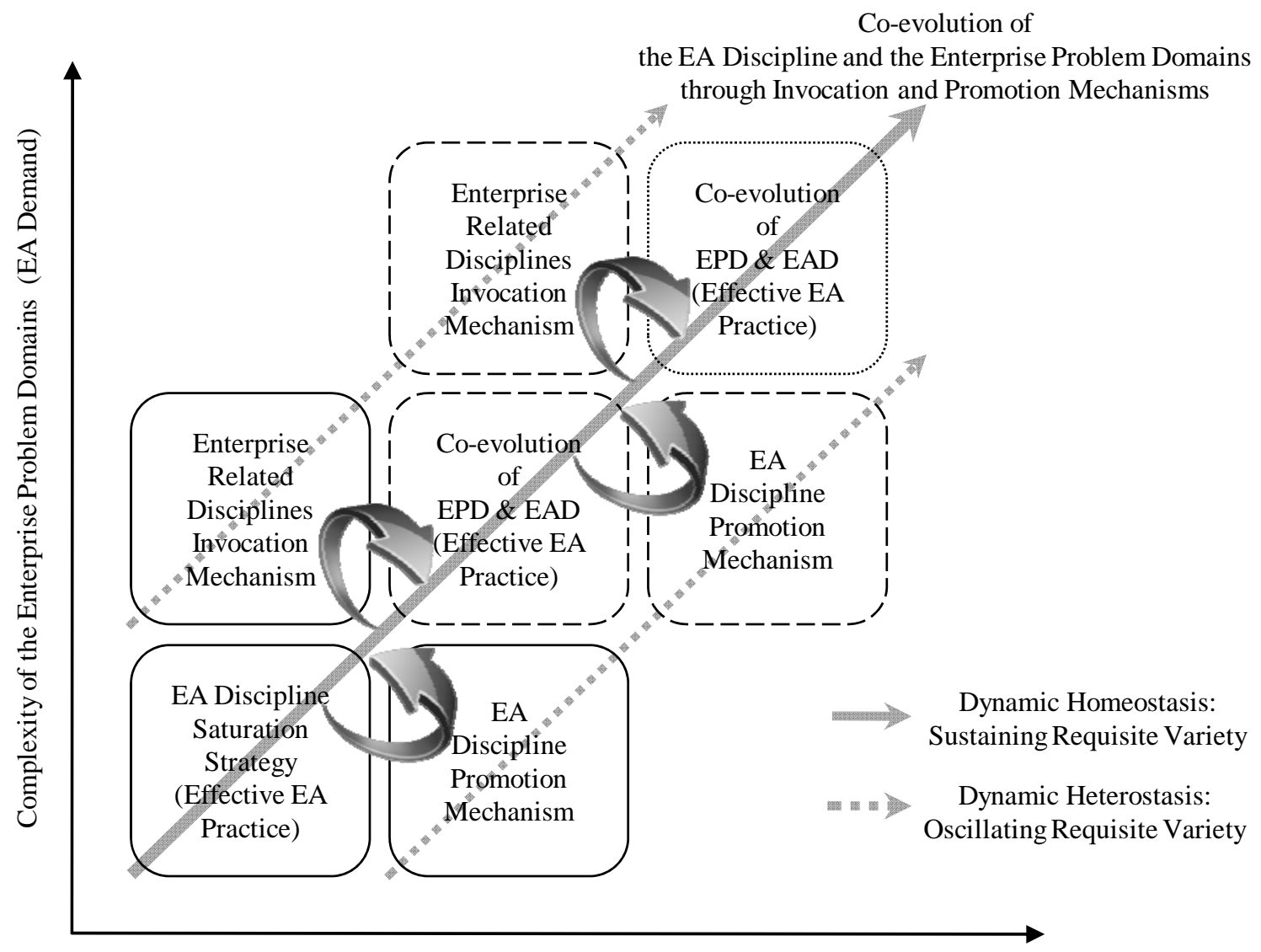

Complexity of the Enterprise Architecture Discipline (EA Supply)

Figure 5. Co-evolution Path Model of the Enterprise Architecture Discipline

Once we developed such theories, we can state problems - not only existing problems in concrete problem domains, but also formalised, harmonised and synthesised problem statements by EA cybernetics within this new theory. Based on a new theory, one could express new problems and find new solutions / models never before contemplated.

A unified cybernetic theory of EA may be used to develop a corresponding methodology (or rather, methodologies) for use in EA practice. Results of such synthesis must be tested in practice (through intervention, influence, or observation) to create 'case records', which in turn provide the source of criticism which allow better theories to be formulated (and as a result, better models, techniques, and methodologies). The application of the latter methodologies should be documented in 
case records which could provide feedback to improve the individual- and the unified theories.

The EA discipline not only embraces models, methods and theories of management and control it also uses the same from systems engineering, linguistics, cognitive science, environmental science, biology, social science and artificial intelligence.

What cybernetic thinking is able to do is to provide a method of unifying (and relating) the apport of these disciplines: cybernetic thinking can be used to represent the essence of multiple theories using abstract functions and processes (and metaprocesses) and their relationships / rules / axioms (likely to be expressed in suitably selected logics).

Following the systems thinking diagram of Fig.6., the contributions of these disciplines needs to be formalised, synthetised, harmonised, systematised and eventually represented as a unified Cybernetic Theory of EA (which we call 'EA Cybernetics').

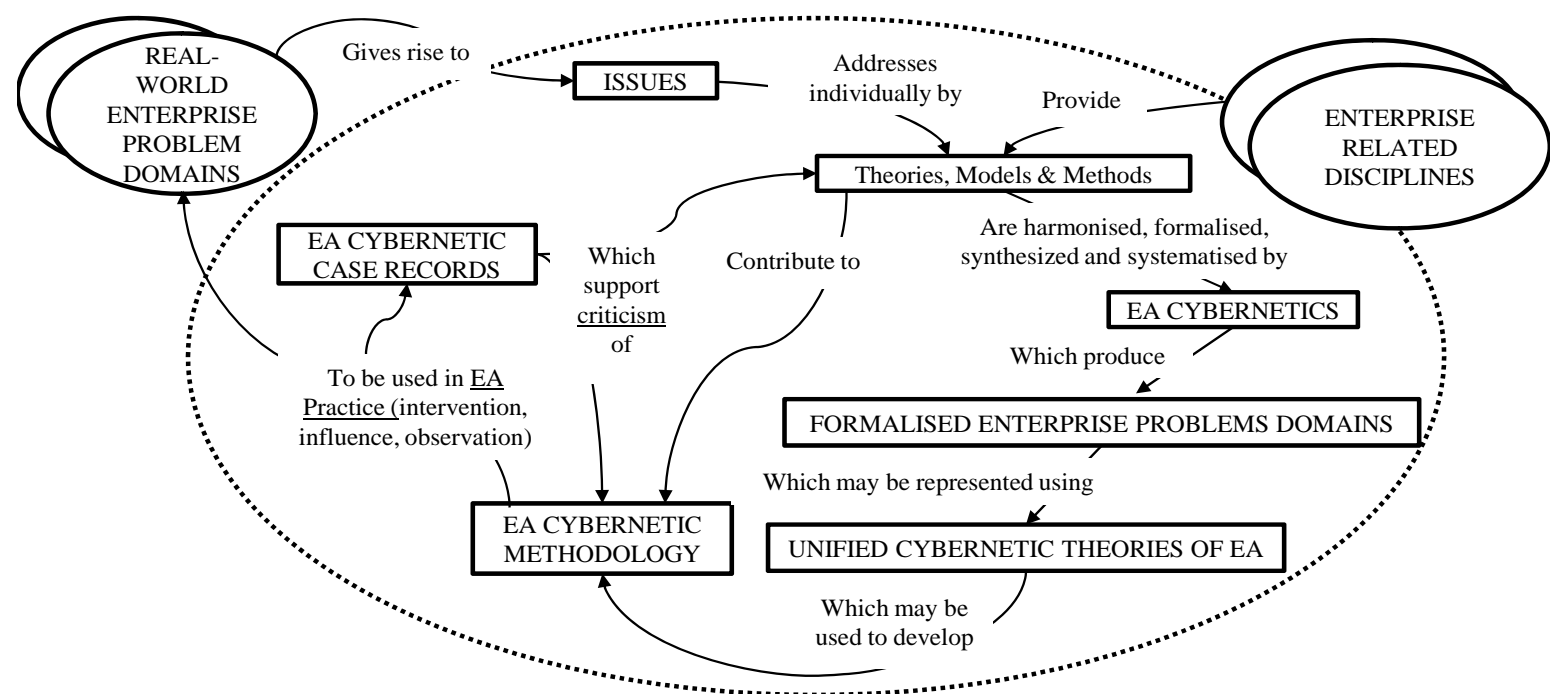

Figure 6. A Cybernetic Model of Enterprise Architecture Discipline as a Developing Discipline based on the relationship between activities and results in a developing discipline (after Anderton and Checkland 1977; Checkland 1996)

\section{CONCLUSIONS}

In order to have an evolving unified theory to extend the EA Body of Knowledge with common elements that are independent from the domain and the type of change, we focused on the viability of EA as a discipline and discussed it using Beer's Viable System Model (VSM), and correspondingly introduced three basic components of a viable EA discipline using VSM.

We also proposed the concept of a co-evolution mechanisms for an evolving EA discipline based on VSM and a companion theory (Ashby's law of requisite variety, but with a new, refined definition of the complexity measure for the model(s) of the environment, that takes the relativity of this term into account).

We also proposed a cybernetic model of EA as a developing discipline using Checkland's system model for a developing discipline and introduced EA Cybernetics as a distinct field of EA that harmonises, formalises, synthesises and systematises the results of systems thinking and cybernetics to enable their concerted application in EA practice.

Future work will concentrate on the application of this model of EA as an evolving discipline, whereupon testing and validation of this theoretical model is to be performed. 


\section{REFERENCES}

Anderton, R. H. and P. B. Checkland (1977) On learning our lessons. Internal Discussion Paper. Lancaster, UK, Department of Systems, University of Lancaster. 2/77

Ashby, W. R. (1940) Adaptiveness and equilibrium. The British Journal of Psychiatry 86(362) pp478-483

Ashby, W. R. (1956) An introduction to cybernetics. London : Chapman \& Hall

Ashby, W. R. (1960) Design for a brain; the origin of adaptive behavior. New York : Wiley

Beer, S. (1966) Decision and Control: The Meaning of Operational Research and Management Cybernetics. New York : Wiley

Beer, S. (1979) The Heart of Enterprise: the Managerial Cybernetics of Organization. New York : Wiley

Beer, S. (1981) Brain of the Firm, 2nd Ed. New York : Wiley

Beer, S. (1985) Diagnosing the system for organizations. New York : Wiley

Bertalanffy, L. (1968). General System TheoryFoundations and Developments. New York: George Braziller, Inc, 10.

Bødker, K., Kensing, F., \& Simonsen, J. (2004). Participatory IT design: designing for business and workplace realities: The MIT Press.

Boulding, K. E. (1956) General systems theory-the skeleton of science. Management Science 2(3) pp197208

Checkland, P. (1996) Systems Thinking, Systems Practice. Chichester, UK, John Wiley \& Sons.

Conant, R. C. and W. R. Ashby (1970). Every Good Regulator of a System Must be a Model of That System. International Journal of Systems Science 1(2): 89-97

Doumeingts, G. (1984). La Methode GRAI [PhD Thesis]. Bordeaux, France: University of Bordeaux I.

Doumeingts, G. (1998). GIM, Grain Integrated Methodology. In A. Molina and A. Kusiak and J. Sanchez (Eds.), Handbook of Life Cycle Engineering, Models and Methodologies (pp. 227-288). Dordrecht: Kluwer Academic Publishers

DoDAF (2009) DoD Architecture Framework Version 2.0, US DoD, Washington.

Gell-Mann, M. (1994) Complex adaptive systems. in Complexity: Metaphors, models, and reality. G.A. Cowan,. D. Pines, and D. Meltzer (Eds) Reading, MA : Addison-Wesley. pp. 17-45.

Geoghegan, M. C. and P. Pangaro (2009). Design for a self-regenerating organisation. International Journal of General Systems 38(2): 155-173

Hammer, M., \& Stanton, S. A. (1995). The reengineering revolution: A handbook: HarperBusiness New York.

Holland, J. H. (1992). Complex adaptive systems. Daedalus 121(1): 17-30.

IFIP-IFAC-Task-Force (1999). GERAM: Generalised enterprise reference architecture and methodology. Version 1(3): 6-3.

ISO15704 (2000, Amd.2005). Industrial automation systems -- Requirements for enterprise-reference architectures and methodologies. Geneva : ISO TC184.SC5.WG1. Geneva, ISO TC184.SC5.WG1.

ISO/IEC/IEEE 42010 (2011) Systems and software engineering - Architecture description, Recommended Practice for Architectural Description of Softwareintensive Systems. ISO/IEC JTC1/SC7/WG42.

Kandjani, H., Bernus, P. (2011) Engineering SelfDesigning Enterprises as Complex Systems Using Extended Axiomatic Design Theory. Proc of the 18th IFAC World Congr. Miloan, Italy, IFAC Papers On Line, 18(1). Amsterdam : Elsevier, 11943-11948

Kensing, F., Simonsen, J., \& Bodker, K. (1998). MUST: A method for participatory design. Human-Computer Interaction, 13(2), 167-198.

Nonaka, I; Takeuchi, H (1995). The knowledge creating company: how Japanese companies create the dynamics of innovation. New York : Oxford University Press.

Pask, G. (1975) Conversation, Cognition and Learning. Amsterdam : Elsevier

Senge, P. M. (1993). The Fifth Discipline: The Art and Practice of the Learning Organization: Book review.

Suh, N.P. (1990) The Principles of Design. New York : Oxford University Press

Suh, N.P. (2001) Axiomatic design: advances and applications. New York : Oxford University Press.

Suh, N. P. (2005) Complexity: Theory and Applications. New York : Oxford University Press

TOGAF (1999-2011) TOGAF 1 - TOGAF 9.1 (Versions of the TOGAF Architecture Framework). Open Group

Umpleby, S. A. (2009). Ross Ashby's general theory of adaptive systems. International Journal of General Systems 38(2): 231-238.

Wiener, N. (1948) Cybernetics or Control and Communication in the Animal and the Machine. (2nd Rev. Ed 1961). Cambridge, MA : MIT Press

Wooldridge, M. J. (2002). An introduction to multiagent systems : Wiley. 